\title{
Insegurança alimentar e estado nutricional de famílias beneficiárias do Programa Bolsa Família
}

\author{
Carina Aparecida Pinto, Dayane de Castro Morais, Lucimar Aguiar da Silva, Sylvia do Carmo \\ Castro Franceschini, Silvia Eloiza Priore
}

\section{Resumo}

Segurança alimentar e nutricional caracteriza-se pelo acesso permanente a alimentos de qualidade e em quantidade suficiente para todas as pessoas, sem comprometer o acesso a outras necessidades básicas. Objetivou-se neste estudo verificar relação entre a situação de (in) segurança alimentar e o estado nutricional de famílias beneficiárias do Programa Bolsa Família (PBF). Estudo transversal realizado com 206 famílias beneficiárias do PBF residentes na zona urbana do município de Viçosa, Minas Gerais. Avaliou-se a situação de insegurança alimentar pela Escala Brasileira de Insegurança Alimentar (EBIA). Para avaliar o estado nutricional de crianças e adolescentes foram observados os valores em z escore, segundo sexo, dos índices Estatura/Idade e IMC/Idade (WHO 2006; 2007); para adultos e idosos IMC, segundo WHO (1998) e Lipschitz (1994), respectivamente e para gestantes IMC/semana gestacional de acordo com Atalah (1997). Avaliou-se estatura de adultos e idosos com a finalidade de verificar presença de desnutrição pregressa também nestas faixas etárias. Utilizou-se a curva da WHO (2007) e ponto de corte menor que -2 z escore para baixa estatura, com os indivíduos aos 19 anos, pois ao final da adolescência o indivíduo já tem seu crescimento finalizado. Aferiu-se o perímetro da cintura (PC) de adolescentes, adultos e idosos durante a expiração normal, na cicatriz umbilical. Com esta medida e a de estatura calculou-se a relação cintura estatura (RCE), considerando a presença de risco cardiometabólico valores de RCE $\geq 0,50$, independentemente do sexo e idade. As famílias foram classificadas como em insegurança alimentar e nutricional, pelo estado nutricional, quando pelo menos um de seus integrantes apresentava baixa estatura, baixo peso, excesso de peso (sobrepeso e/ou obesidade) ou risco cardiometabólico. O estudo foi aprovado pelo Comitê de Ética em Pesquisa com seres humanos da Universidade Federal de Viçosa, com número de parecer 691.641/2014 e a participação dos voluntários ocorreu mediante assinatura do Termo de Consentimento Livre e Esclarecido e Termo de Assentimento. Realizou-se estatística descritiva dos dados e verificou-se associações entre (in) segurança alimentar e estado nutricional da família pelo teste de qui-quadrado de Pearson. A magnitude da associação foi determinada pela Odds Ratio (OR; IC95\%). Adotou-se nível de significância menor que 0,05. Pela EBIA detectou-se prevalência de $82,0 \%$ de insegurança alimentar nas famílias, sendo que desta, 56,8\% apresentaram insegurança alimentar leve, 17,9\% moderada e 7,3\% grave. Em relação ao estado nutricional, não observou-se associação entre insegurança alimentar e presença de pelo menos um integrante da família com baixa estatura, baixo peso, sobrepeso, obesidade ou risco cardiometabólico ( $p>0,05)$. No entanto, a presença de pelo menos um integrante com distrofia nutricional (baixa estatura, baixo peso ou excesso de peso) ou risco cardiometabólico, esteve presente em $78,7 \%$ e $86,8 \%$ das famílias em insegurança alimentar, respectivamente. Famílias beneficiárias do PBF constituem público com menor renda e em maior vulnerabilidade social, portanto mais susceptíveis às condições de insegurança alimentar, sendo importante a análise dos fatores associados a (in) segurança alimentar e nutricional. Pois, a EBIA avalia a insegurança alimentar, sendo necessária a avaliação do estado nutricional e do risco cardiometabólico que abrangem a dimensão nutricional.

Descritores: Segurança Alimentar; Estado Nutricional; Programa Bolsa Família. 